\title{
Referencias pedagógicas de la educación expandida*
}

\author{
Alejandro Uribe Zapata ${ }^{* *}$ \\ Recibido: 25 de mayo del 2018 \\ Enviado a pares evaluadores: 19 de junio del 2018 \\ Aprobado por Comité editorial: 29 de junio del 2018 \\ https://doi.org/10.22395/csye.v7n13a5
}

\section{RESUMEN}

Este artículo analiza algunas referencias pedagógicas asociadas al término educación expandida, tales como: educación alternativa, pedagogías críticas y pedagogías emergentes, con el propósito de precisar dichos términos y explorar su relación con el concepto y las prácticas de educación expandida. Se estudian tanto las semejanzas como las disimilitudes de los términos y se concluye que: 1) la educación alternativa influye de manera directa en muchas de las prácticas de la educación expandida; 2) la educación expandida presenta una cercanía con las pedagogías críticas; y 3) en contravía de la visión imperante que considera que la educación expandida es una pedagogía emergente, se demuestra que esta es en realidad un discurso crítico/práctico sobre educación, sin compromiso normativo ni prescriptivo.

Palabras clave: tecnología educativa; educación alternativa; pedagogía; historia de la educación; (tesauro); educación expandida; pedagogías emergentes.

\footnotetext{
* Este artículo de reflexión es una versión resumida de uno de los capítulos que hacen parte del trabajo El hilo de Ariadna: concepto y prácticas de educación expandida realizado en el marco del Doctorado en Educación, línea de investigación Educación y TIC de la Universidad de Antioquia.

** Magíster en Educación de la Universidad de Antioquia (Colombia) y filósofo de la misma universidad, en la cual se encuentra cursando el Doctorado en Educación. Correo electrónico: alurizap@gmail.com. Orcid: http://orcid. org/0000-0002-9228-9088
} 


\section{Expanded Education Pedagogical References}

\section{ABSTRACT}

This article analyzes some pedagogical references associated with the term expanded education, such as: alternative education, critical pedagogies and emerging pedagogies, in order to specify such terms and explore their relationship with the concept and practices of expanded education. Term similarities and differences are studied and it is concluded that: 1) alternative education directly influences many practices of expanded education; 2 ) expanded education presents convergence in many aspects with critical pedagogies; and 3), contrary to the prevailing idea that expanded education is an emerging pedagogy, it is shown that it is actually a critical and practical discourse on education, with no normative or prescriptive commitment.

Keywords: educational technology; alternative education; pedagogy; history of education (thesaurus); expanded education; emerging pedagogies.

\section{Referências pedagógicas da educação expandida}

\section{RESUMO}

Este artigo analisa algumas referências pedagógicas associadas ao termo educação expandida, tais como: educação alternativa, pedagogias críticas e pedagogias emergentes, com o propósito de determinar tais termos e explorar sua relação com o conceito e com as práticas de educação expandida. Estudam-se tanto as semelhanças quanto as diferenças dos termos e conclui-se que: 1) a educação alternativa influencia de maneira direta em muitas das práticas da educação expandida; 2) a educação expandida apresenta uma proximidade com as pedagogias críticas; e 3) ao contrário da visão que impera e considera que a educação expandida é uma pedagogia emergente, demonstra-se que ela é, na realidade, um discurso crítico/ prático sobre educação, sem compromisso normativo nem prescritivo.

Palavras-chave: tecnologia educativa; educação alternativa; pedagogia; história da educação; (tesauro); educação expandida; pedagogias emergentes. 


\section{Introducción}

Este artículo explora algunos referentes pedagógicos asociados al término educación expandida. Los términos escogidos derivan de algunas definiciones asociadas a este concepto emergente y son los siguientes: educación alternativa, pedagogías críticas y pedagogías emergentes. A continuación se pretende precisar dichos términos y explorar su relación con el concepto y las prácticas de educación expandida.

Tanto en el caso del concepto como en el de las prácticas de educación expandida, no es posible identificar un momento de emergencia concreto ni un corpus de ideas claramente delimitado y organizado al cual se asocien. Al contrario, se alimentan de una amalgama de ideas y prácticas provenientes de diversos campos. No obstante, se caracterizan por: considerar aspectos conceptuales y metodológicos de la educación no formal e informal, y de la cultura digital; estimular relaciones inter y transdisciplinares; y estar disponibles para la ciudadanía, es decir que son participativas, experienciales y procesuales (Uribe Zapata, 2018).

Con la finalidad de presentar una síntesis, este artículo se concentra en tres referentes pedagógicos para explorar la semejanza y disimilitud que presentan con respecto al término educación expandida ${ }^{1}$. Estos se seleccionaron a partir de puntos clave de las definiciones de Freire (2012), Fonseca Díaz (2012), Díaz López $(2009,2012)$ y Díaz, Gil y Jiménez (2010).

Por referentes pedagógicos se entienden aquellos insumos teóricos con un carácter más endógeno, propios de la institucionalidad, y que se emplean -sin tapujos-así su uso no sea homogéneo. Se hace entonces mención a tres términos: educación alternativa, pedagogías críticas y pedagogías emergentes. Como se explicó, el objetivo es precisar los términos y ver su relación con el concepto y las prácticas de la educación expandida.

\section{Educación alternativa}

Según la Enciclopedia Británica (Encyclopaedia Britannica, 2009), la educación alternativa es aquella que difiere de la que ofrecen las escuelas convencionales ${ }^{2}$. Es posible encontrar ejemplos tanto dentro del sistema escolar como por fuera del mismo, el carácter alternativo puede estar en el mobiliario, en los temas a trabajar, en la organización curricular o en el tipo de relaciones que se fomentan entre docentes y estudiantes. Así, en la categoría caben las escuelas tipo

\footnotetext{
1 No son los únicos referentes. Cercanos a estos están los de educación no formal e informal, teorías de la desescolarización, aprendizaje social, estudios de comunicación y educación, entre otros (Uribe Zapata, 2017a)

2 En España, por ejemplo, tienen un buscador específico para proyectos que abogan por esta idea. Véase http:// ludus.org.es/es
} 
Summerhill, las aulas abiertas que se popularizaron en los Estados Unidos a partir de la década de 1970, el pensamiento pedagógico de la escuela Nueva o el movimiento crítico, hasta la educación en casa (homeschooling) ${ }^{3}$ y las escuelas que Rosan Bosch y su equipo diseñaron (Rosan Bosch, s.f.).

Al ser la educación alternativa una reacción a la educación obligatoria estandarizada, repetitiva y reproductora del statu quo presente en diversos momentos históricos incluyendo el actual, lo alternativo ha adquirido múltiples matices con el paso del tiempo. Por ejemplo, durante la Ilustración y la puesta en marcha de los ideales suscritos por la Revolución Francesa, lo alternativo eran ideas como las de Rousseau que promovían la libertad de pensamiento y la no atadura a los condicionamientos sociales, particularmente el yugo de los reyes absolutistas y la vertiente déspota del clero. Antes que controlar a la sociedad civil a través de enseñanza pública obligatoria, Rousseau buscaba el desarrollo libre y espontáneo de las personas, primero a través de la experiencia y posteriormente vía la educación. Por esta, y otras posturas pedagógicas revolucionarias en aquel entonces, Rousseau es considerado el precursor intelectual de la Escuela Nueva (Godotti, 1998)

A finales del siglo XIX e inicios del siglo XX se gestó dicho movimiento de renovación escolar, también llamado de otras formas (pedagogía progresista, escuela activa, etc.) y fue el más influyente de los últimos años. Aunque este no es el espacio para reseñar a cada una de las figuras que han desempeñado un papel en la configuración de esta escuela, cabe mencionar a Ferrière, Montessori, Decroly, entre otros; es importante subrayar algunos rasgos generales, independientemente de cómo fueron llevados a la práctica, su país de origen o cómo se ajustaron a los enfoques teóricos de cada pensador. En este caso, se van a subrayar algunos elementos de esta iniciativa, gestada desde el propio seno del sistema, ya que es allí donde se pueden vislumbrar con detalle aspectos pedagógicos que todavía siguen presentes en diversas prácticas educativas expandidas.

Para dilucidar tales principios, hay dos documentos oficiales que sintetizan las metas y aspiraciones de la Escuela Nueva (Marín Ibañez, 1976). El primero, titulado Liga Internacional de la Educación Nueva, tiene siete (7) principios; el segundo, titulado Oficina Internacional de las Escuelas Nuevas, tiene treinta (30). Con estos documentos se buscaba depurar la creciente multiplicación de escuelas que solo aprovechaban la popularidad del término para adherirse al movimiento, pero sin alterar sus propias realidades educativas. No obstante, esto resultó ser un arma de doble filo. Por ejemplo, como dato curioso, pero también ilustrador, solo una escuela cumplía con todos los principios y varias escuelas

3 En Colombia, por ejemplo, existe la Red Colombiana de Educación en Familia. Véase http://www.enfamilia.co/ 
de renombre difícilmente superaban el umbral mínimo de 15 condiciones que había propuesto el mismo Ferrière, promotor de esta iniciativa.

Al sintetizar estos principios, se pueden rescatar las ocho ideas siguientes (Godotti, 1998; Marín Ibañez, 1976; Trilla Bernet, 2001).

- El carácter renovador, de apertura y alternativo de estas escuelas en contraposición con aquellas memorísticas, repetitivas, transmisoras, preocupadas solo por la disciplina y por cumplir un currículo.

- Su ubicación geográfica en el campo, para así tener contacto con la naturaleza, y su carácter de internado, para emular -en parte- los códigos familiares y la coeducación.

- En sintonía con lo anterior, una defensa de lo natural, artesanal y experiencial; se pregona un contacto no mediatizado, sobre todo en los primeros años.

- Acento en ciertas habilidades mentales que resultan claves para acercarse al mundo social y natural, tales como la observación, la elaboración de preguntas, las hipótesis de trabajo, entre otras asociadas a la elucubración intelectual, antes que a los contenidos o la mera adquisición de datos enciclopédicos.

- Defensa por la formación y el cultivo del cuerpo a través de los deportes y la gimnasia, en suma, el reconocimiento de la importancia que tiene la educación física.

- Un paidocentrismo acentuado, en el que todos los componentes educativos se ponen al servicio del aprendiz al tiempo que se fomenta la actividad del estudiante como principal forma de aprendizaje.

- Fomento del autogobierno escolar fundado en una educación que aboga por la libertad y la participación conjunta de los estudiantes.

- Promoción de la educación artística, se le deja de considerar simplemente como un asunto menor o accesorio de las otras disciplinas.

Para cerrar este aparte, vale la pena subrayar al menos una propuesta pedagógica alternativa del siglo XX promovida desde la educación escolar: la Escuela Moderna de Freinet ${ }^{4}$.

4 Como toda elección, esta es arbitraria y caprichosa ya que no incluye pensadores de la talla de Montessori y sus materiales (incluyendo mobiliario) para estimular el pensamiento de los niños; Decroly y los centros de interés; o Sutherland Neill y la Escuela de Summerhill, entre otros. Para una síntesis global de movimientos pedagógicos del siglo XX, véase la obra coordinada por Trilla Bernet (2001). 
Este pedagogo francés no reflexionaba sobre la escuela a partir de postulados teóricos únicamente, él trabajaba en una como maestro. El dato biográfico importa, ya que si bien fue un lector de la tradición renovadora clásica (Montaigne, Rousseau o Pestalozzi) y las diversas corrientes de la Escuela Nueva (Dewey, Decroly, Montessori, entre otros), criticó con dureza el idealismo y el escaso correlato práctico de estas propuestas. De ahí que sus reflexiones pedagógicas fueran fruto de su propio quehacer diario y que se mostrara renuente a considerar sus ideas como un método, esto es, un grupo de conocimientos fijos, rígidos, inmunes al contexto y aplicables, como si fueran una receta de cocina. Al contrario, por su mutación, capacidad de adaptación a las diversas situaciones y su carácter parcial e inacabado (González Monteagudo, 1988), prefería el término técnicas, ya que estas se legitiman o descartan en la práctica pedagógica y en contextos escolares reales. Apelando a términos de la informática, Freinet era un defensor de las estrategias bottom-up (de abajo hacia arriba) antes que las top-down (de arriba hacia abajo).

Otro dato biográfico de interés fue su participación activa en la Primera Guerra Mundial, ya que las heridas de guerra afectaron su tono de voz, el cual perdió intensidad. En gran medida, por esa limitante física tuvo que recurrir a una serie de recursos adicionales, técnicas, para no ver alterada su práctica pedagógica (Imbernón Muñoz, 2001). Algunas de estas técnicas y recursos adicionales, que se ponían constantemente a prueba en su escuela y adoptadas en el movimiento denominado Escuela Moderna (en honor a Ferrer Guardia), son el texto y dibujo libre, la correspondencia entre escuelas, el diario escolar, la imprenta, el limógrafo, entre otros.

La carta de la Escuela Moderna, un documento que se redactó en 1968, ilustra los diez (10) principios de esta escuela. Por su valor, los trascribo de manera literal según mi propia traducción libre del original en francés (ICEM, 2000):

1. La educación es desarrollo y elevación, no una acumulación de conocimientos, un amaestramiento o una servidumbre.

2. Nos oponemos a todo adoctrinamiento

3. Nos negamos a la ilusión de una educación que es suficiente por ella misma y fuera de las influencias sociales y políticas que la condicionan

4. La escuela del mañana será la escuela del trabajo

5. La escuela del mañana estará centrada en el niño

6. La investigación experimental es la base y la primera condición de nuestro esfuerzo de modernización escolar para la cooperación

- 116 
7. Los educadores que siguen la pedagogía de Freinet son los responsables de la orientación y la extensión de las ideas cooperativas

8. Nuestro movimiento de la Escuela Moderna está preocupado por mantener las relaciones de simpatía y colaboración con todas las organizaciones que trabajen en el mismo sentido.

\section{Nos relacionamos con la Administración}

10. La Pedagogía Freinet es, por esencia, internacional.

Las técnicas de Freinet mencionadas con anterioridad, adicionalmente a su valor didáctico, compartían una serie de principios con la Escuela Nueva que vale la pena destacar. En efecto, valoraban el tanteo experimental, la educación por y para el trabajo, la cooperación entre estudiantes, la importancia del ambiente escolar y social en el que estaba inscrita la escuela y la necesidad de crear materiales puntuales para así potenciar las ideas, pero desde el plano de la práctica educativa (Freinet, 1996).

En términos más filosóficos, Freinet rechazaba todo lo formal (él lo llamaba lo escolástico), organizado y artificial, y defendía un vitalismo que se alimentaba de la misma práctica pedagógica. La pedagogía no solo se debía nutrir del ensayo y el error, sino que debería servir para generar cambios sociales e intelectuales, pero desde espacios reales de trabajo y no a partir de discursos lejanos o textos abstractos sin correlato en la práctica (Legrand, 1993). En ese sentido, las ideas pedagógicas de Freinet anticipan parte de los discursos asociados a la cultura del hacer y se alimentan de un matiz político explícito.

\section{Relación de la educación alternativa con la educación expandida}

La educación expandida se alimenta del ecosistema de ideas que se pueden catalogar dentro de la difusa, pero amplia etiqueta de educación alternativa. A continuación un par de ejemplos para exponer esta idea.

La educación expandida tiene estrecha relación con la cultura digital. Y dentro de esta cultura, uno de los fenómenos más llamativos de los últimos años ha sido la transición de la web 1.0 a la web 2.0. El cambio no fue solamente un asunto tecnológico, sino también social y cultural. El nuevo escenario permitía el consumo y la lectura pasiva de contenidos -como antaño- y además ampliaba las posibilidades creativas de los usuarios en términos de escritura y producción. En el plano educativo, una vieja aspiración de las pedagogías alternativas y activas empezaba a cobrar vigencia: que el estudiante fuese el protagonista, puesto que tiene recursos a su disposición para expresarse a su gusto y la nueva y amplia gama de posibilidades que ofrece este entorno suplen 
las posibles limitaciones técnicas del docente, de ahí que este adquiera otro rol (Prats, 2013). En la educación expandida el centro es el aprendizaje y por tanto, el sujeto que aprende.

Un segundo punto se asienta en la cultura del hacer, defendida por Dewey a través de su aprendizaje a partir de la experiencia, o para decirlo en términos de ahora, el aprender haciendo; o por Freinet gracias a su defensa del tanteo experimental y las prácticas concretas en espacios de aula por encima de las deliberaciones teóricas fuera de contexto. Estas propuestas pedagógicas alternativas tienen unos antecedentes interesantes en términos educativos, así rara vez sean transparentes en su formulación. No obstante, no es simplemente una recuperación actual de una idea de antaño, mucho menos un hacer por el hacer. Hoy en día se apela a este enfoque, no solo por su supuesta potencialidad pedagógica, sino porque también es un ejercicio que invita a la apropiación, la participación y reflexión sobre los objetos que nos rodean, configuran e inciden de manera exponencial en nuestras prácticas cotidianas. En suma, en la educación expandida se trata de exprimir la idea que hacer es pensar (Sennett, 2009).

\section{Las pedagogías críticas}

Desde la década del setenta del siglo pasado empezaron a emerger propuestas críticas interinas que no solo entendían a los sujetos como agentes de cambio y de transformación social, sino que tenían en cuenta las complejidades propias de las instituciones escolares y se enfrentaban a temáticas tabú tales como el género, la raza o las preferencias sexuales en contextos escolares.

Las pedagogías críticas, como todas las ideas sugerentes en la esfera educativa, no se configuran a partir de un conjunto homogéneo de ideas sino que se alimentan a partir de múltiples fuentes teóricas y asumen diversas formas desde la práctica. Con todo, emerge -con regularidad- la vocación por vincular la educación con la situación social imperante, tal es el caso de Paulo Freire.

Tal como hizo Dewey (2004) en su momento, Freire difuminó las distancias entre sujeto y objeto al asumir una dialéctica permanente entre extremos conceptuales y prácticos. También asumió la pedagogía como una práctica política atada al contexto y no como un recetario metodológico que se sigue paso a paso de manera acrítica. Por ello, vale la pena prestar atención a dos ideas sugerentes, entrelazadas e inseparables que permean todo el corpus de la obra de Freire, a saber, la concientización y la educación libertaria.

La concientización, o concienciación -respetando la terminología original de Freire- es el esfuerzo liberador por el que pasa el oprimido para arribar a una toma de conciencia. Es un despertar que incluye tres fases que no se siguen 
necesariamente de manera lineal y menos al pie de la letra: la mágica, la ingenua y la crítica. En la primera hay impotencia y resignación, por lo que no se hace nada para cambiar la situación. En la segunda se reconocen los problemas a un nivel individual, pero todavía no se entienden las causas de los mismos plenamente, de ahí que las acciones no sean congruentes y asuman también el rol del opresor. En la tercera se entiende mejor la estructura opresiva y se logran entender los problemas, pero en función de comunidad, por lo que las acciones subsiguientes, en el diálogo con otros, pretenden cambiar ese estado de cosas ( Freire, 2011).

La educación libertaria es la antípoda de la que Freire llama "educación bancaria", esta es esa educación domesticadora en la que el educador se limita a depositar de manera unidireccional una información estática en las mentes pasivas, acríticas y anestesiadas de los educandos, pero sin posibilidad de réplica, sin considerar su experiencia previa y en el fondo buscando que se adecúen sin rechistar, al orden social establecido. La educación liberadora, por su parte, problematiza la realidad, no la asume como algo dado que hay que aceptar, sino como un punto de partida susceptible de cambio, y establece un diálogo constructivo entre el educador y el educando que busca transformar la realidad. Antes que adaptarse a la realidad, los sujetos la recrean, la enriquecen y actúan en ella gracias a la interacción y al diálogo activo con los otros. Tal como la alfabetización de los campesinos adultos de Freire, constituye un acto político, ya que no busca enseñar simplemente a decodificar de manera mecánica el código escrito e incluye la lectura de los propios contextos. Para resumir este pasaje, es mejor apelar a las propias palabras de Freire:

Lo importante, desde el punto de vista de la educación liberadora y no "bancaria", es que, en cualquiera de los casos, los hombres se sientan sujetos de su pensar, discutiendo su pensar, su propia visión del mundo, manifestada, implícita o explícitamente, en sus sugerencias y en las de sus compañeros. (Freire, 2005, p. 158)

Después de Freire, varios autores siguieron configurando y enriqueciendo la compleja cartografía de las pedagogías críticas, pero con matices. Por ejemplo, Peter McLaren, pedagogo canadiense, habla de Freire como su mentor intelectual y su propuesta de pedagogía crítica revolucionaria es casi una extensión de las ideas del pedagogo brasilero. Otros, como los pedagogos norteamericanos Henry Giroux y Michael Apple, se nutren de una amplia diversidad de tradiciones teóricas, tales como Marx, el neomarxismo cultural de Gramsci, la escuela crítica de Frankfurt y su segunda generación, en particular de Habermas.

¿Cuáles son los elementos comunes en las obras de estos autores y otros que no se mencionan por falta de espacio? Vamos a seguir cuatro de las cinco señas de identidad que rescata Carbonell Sebarroja (2015). Primero, un paso de la crítica 
a la transformación. No solo se analizan las diversas y complejas relaciones de poder, así como las tensiones internas en las instituciones educativas, sino que se abre un espacio de posibilidad para la emancipación transformadora a través del reconocimiento de los sujetos como seres activos.

Segundo, una apuesta contra la neutralidad, el pensamiento único y la fragmentación curricular. Acá la mira está puesta en la organización y selección de contenidos que configuran el currículo, explícito y oculto, ya que este rara vez es neutral, suele ser el fruto de intereses e ideologías ajenas a la estructura escolar que terminan condicionando la socialización de los estudiantes. Un currículo acorde, entonces, debe al menos respetar la amplia diversidad cultural y social e incluir así las voces marginadas; evitar los contenidos ahistóricos y acontextuales, conectar con el entorno y la memoria local; y promover una integración de saberes antes que una fragmentación curricular.

Tercero, el diálogo y la cultura cruzan los muros del aula. Los procesos de emancipación, así como las dinámicas dialógicas, no se presentan solo en contextos de aula sino también por fuera de ella. En ese orden, una escuela pública es aquella que se vincula activamente con su comunidad y no exclusivamente con lo estatal o lo corporativo. Desde esta perspectiva, las distancias entre la escuela y el mundo de la vida se minimizan al máximo.

Cuarto, el profesor como intelectual transformador. El educador no es visto como un mero técnico, un burócrata acomodado o solamente como un transmisor de ideas ajenas, sino como una persona comprometida con la crítica, la acción y los procesos de liberación y emancipación. En esta línea, también se crítica la figura del intelectual aislado de la realidad y sin ningún tipo de función social. Al contrario, los educadores deben ser personas comprometidas, una idea que puede desembocar en muchos casos en el activismo y la militancia. Así se vislumbra el reto mayor: "conseguir que lo pedagógico sea más político y lo político más pedagógico" (Carbonell Sebarroja, 2015).

\section{Relación de las pedagogías críticas con la educación expandida}

En efecto, la emergencia de conceptos y prácticas asociadas a la educación expandida es de por sí una alteración al estado de cosas imperante y una crítica a la gramática de la modernidad. Por ejemplo, al ofrecer rutas formativas desligadas de la institucionalidad escolar, así como formas alternativas de aprendizaje, es posible estimular la conciencia crítica al ver que es posible elaborar otros discursos alrededor de la educación en particular, y la modernidad en general. Además, las prácticas educativas expandidas, al ser sectorizadas, comunales y estar atadas a contextos específicos, promueven, no siempre de manera consciente, dinámicas políticas de carácter molecular y ejercicios de 
glocalidad circunscritos a coordenadas espaciales puntuales, pero motivados por temáticas trasnacionales. O lo que es igual, ponen en práctica dinámicas educativas liberadoras, activistas y de corte político en micro espacios ciudadanos en los cuales es posible la réplica, el diálogo respetuoso entre pares, la problematización de la sociedad y las críticas pacíficas al orden social establecido (Uribe Zapata, 2017b).

\section{Pedagogías emergentes}

Las pedagogías emergentes son,

[...] el conjunto de enfoques e ideas pedagógicas, todavía no bien sistematizadas, que surgen alrededor del uso de las TIC en educación y que intentan aprovechar todo su potencial comunicativo, informacional, colaborativo, interactivo, creativo e innovador en el marco de una nueva cultura del aprendizaje. (Adell Segura y Castañeda Quintero, 2012, p. 15)

Estas pedagogías están vinculadas al concepto de tecnologías emergentes en el contexto educativo, tal como las defiende Veletsianos (2010):

Las tecnologías emergentes son herramientas, conceptos, innovaciones y avances utilizados en diversos contextos educativos al servicio de diversos propósitos relacionados con la educación. Además, propongo que las tecnologías emergentes ("nuevas" y "viejas") son organismos en evolución que experimentan ciclos de sobreexpectación y, al tiempo que son potencialmente disruptivas, todavía no han sido completamente comprendidas ni tampoco suficientemente investigadas. (pp. 3-4)

Así, las pedagogías emergentes se caracterizan por ser tanto ideas nuevas como propuestas pasadas que encuentran un terreno propicio para ser puestas en práctica en el contexto actual. Además, presentan características que no han sido prefijadas de antemano, estas van mutando según el contexto, ya que apelan a estrategias horizontales de aprendizaje entre pares y de difusión de abajo hacia arriba. Al igual que las tecnologías, estas pedagogías también se exponen a ciclos de expectativas desbordadas debido a que se exagera su potencial transformador, pero no por eso se deja de apreciar su potencial disruptivo. Finalmente, extrapolando lo expuesto por Veletsianos, estas pedagogías no han sido completamente comprendidas ni tampoco suficientemente investigadas.

Se subrayan cinco rasgos comunes de este tipo de pedagogías (Adell Segura y Castañeda Quintero, 2015):

- No reducen la educación a un asunto de transmisión/adquisición de conocimientos o una suma de habilidades puntuales para satisfacer las demandas del mercado. Al contrario, creen que educarse es tanto formar ciudadanos para una sociedad compleja como la actual, así como formar sujetos críticos y autónomos aún si pertenecen a un colectivo. 
- Se inspiran tanto en teorías pedagógicas de vieja data, como el constructivismo o el aprendizaje basado en proyectos, así como en ideas aparentemente más actuales, como el conectivismo o el aprendizaje crossover. Los nuevos escenarios de formación estimulan, por una parte, la puesta en práctica de viejos ideales que antes eran inviables, costosos o dispendiosos, pero por otra, generan dinámicas que invitan a la reflexión, puesto que escapan de nuestro sistema categorial clásico.

- Superan los límites espacio/temporales de las aulas escolares, unifican cada vez más los contextos formales de formación con aquellos que no los son, promueven rutas de formación personalizadas y estimulan la creación de entornos de aprendizaje propios según los intereses de cada uno.

- Fomentan conocimientos, habilidades y actitudes asociadas con la metacognición, el aprender a aprender, y lo que en sociología denominan la agencia, esta es, la capacidad que tiene una persona (un agente) de actuar e intervenir sobre el mundo.

- Buscan promover dinámicas formativas que sean al mismo tiempo experiencias significativas y auténticas que apelan al compromiso emocional de los estudiantes, no solo a la dimensión cognitiva. Lo anterior implica la creación de actividades creativas, abiertas y divergentes, asimismo una postura abierta en lo que concierne a la evaluación, ya que no se trata de repetir una serie de contenidos o poner una nota numérica, sino de recrear los insumos, conectarlos con contextos reales y auténticos, y estar atento a la emergencia de aprendizajes no previstos.

En síntesis, las pedagogías emergentes muestran que es posible configurar contratos pedagógicos alternativos en contextos de aprendizaje mediados por lo digital. Si bien en muchos espacios educativos predomina la perspectiva instrumentalizada frente la tecnología o se institucionaliza un uso que refuerza prácticas pedagógicas enfocadas en la transmisión, repetición, vigilancia y control, también empiezan a emerger perspectivas que ven la tecnología como prótesis cognitiva y social que ayuda a transformar el mundo y con ello, la emergencia de diversas dinámicas formativas. No obstante, las pedagogías emergentes todavía son un gerundio, en términos históricos, son muy recientes y deben investigarse más puesto que, así posean un potencial disruptivo según diversas fuentes, podrían generar falsas expectativas.

\section{Relación de las pedagogías emergentes con la educación expandida}

A la luz de lo expuesto, no extraña la proliferación de neologismos, acrónimos y sintagmas de corte educativo que empiezan a reclamar atención, pues parecen conllevar el potencial para crear nuevas formas de intercepción entre la 
pedagogía, la tecnología y el mundo interactivo actual. De hecho, un grupo de investigadores del Instituto de Tecnología Educativa de la Universidad Abierta del Reino Unido, junto con otras entidades, empezaron en 2012 a realizar una serie de informes en los cuales compendian una lista anual de términos, teorías y prácticas con el título de Pedagogía Innovadora (su original en inglés: Innovating Pedagogy). En uno de sus informes más recientes (2015), incluyeron términos como aprendizaje crossover, aprendizaje incidental, pensamiento computacional, aprender haciendo ciencia con laboratorios remotos, entre otros (Sharples et al., 2015).

Por su parte, la investigadora Paola Ricaurte Quijano publicó en su blog un glosario de pedagogías emergentes en el que se encuentran términos tales como aprendizaje ubicuo, pedagogía laparoscópica, educación disruptiva, aprendizaje invisible, aprendizaje en red, pedagogía de pares, entre otros (Ricaurte, 2015). La educación expandida también aparece en ese glosario. Vale preguntarse: ¿es entonces la educación expandida una pedagogía emergente? A mi juicio, no me atrevería a responder esa pregunta de manera afirmativa. Es más, si se entiende la pedagogía desde una perspectiva normativa y no simplemente como un conjunto de saberes descriptivos o explicativos sobre las prácticas educativas, la educación expandida no sería ni pretendería ser una pedagogía.

Para reforzar esto último, vale la pena recordar la distinción realizada entre pedagogías, en el sentido normativo y los discursos sobre educación, a los que no se les exige esa voluntad normativa (Ayuste González y Trilla Bernet, 2005). En ese orden, la educación expandida es más un discurso crítico y contemporáneo sobre la educación antes que una teoría pedagógica que dice cómo debería ser la educación y, más importante aún, cómo hacer para que sea así. La educación expandida no huye de estas preguntas, pero tampoco es su objetivo responderlas como si tuviera la última palabra. Ante todo, no busca prescribir sino provocar y estimular. No sobra recordar que fue promovida por un grupo de curiosos preocupados por la educación, no por un claustro de profesores deseosos de desarrollar un discurso pedagógico nuevo.

Si bien algunos autores defienden de manera bastante convincente la hipótesis de una pedagogía posmoderna de corte normativo (Laudo Castillo, 2011), mi postura en general es escéptica frente los metarrelatos, en este caso, los metarrelatos pedagógicos. En consecuencia, una Pedagogía -en mayúscula-que incluye todas las demás posibles pedagogías -en minúsculas-, no es algo que me seduzca simplemente porque sospecho de esa correlación estrecha entre una sola teoría y múltiples prácticas. Las características pedagógicas de estas iniciativas pueden tener su origen tanto en las pedagogías modernas -ya consolidadas-, como en otras pedagogías, en construcción, no normativas, emergentes y quizás más provocadoras que sugestivas, por citar algunas: pedagogías remix 
(Peña Sánchez et al., 2011), pedagogía líquida (Laudo Castillo, 2015), pedagogía P2P (Fonseca Díaz y Rueda Ortiz, 2012) o paragogía (Fernández Enguita, 2013).

\section{Conclusiones}

A continuación tres conclusiones, una por cada término analizado.

Primero, la educación alternativa influye de manera directa muchas de las prácticas de la educación expandida. En términos pedagógicos, los movimientos de renovación escolar promovidos desde el mismo sistema, enmarcados acá bajo la etiqueta genérica de Escuela Nueva, han influido en las configuraciones formativas que se gestan dentro de estas prácticas.

En concreto, el tránsito desde unas metodologías de aprendizaje centradas en la repetición y recepción pasiva de insumos hacia enfoques pedagógicos emergentes atados al aprender creando, aprendizaje autónomo y capacidad creativa de los estudiantes es muy notorio (Adell Segura y Castañeda Quintero, 2015; Area Moreira, 2015).

Como se infiere de las propuestas mencionadas, la formación deriva de la actividad y de la reflexión sobre la propia experiencia. El aprendizaje se construye de abajo hacia arriba y es un gerundio permanente. De ahí que el énfasis no esté tanto en contenidos curriculares, sino en las destrezas y habilidades que se potencian gracias al proceso experiencial del estudiante, en un marco de libertad, con los diversos artefactos culturales y espacios de formación disponibles en la actualidad.

Es difícil no aceptar que muchos de los principios pedagógicos de la Escuela Nueva están presentes en las configuraciones educativas y formativas de diversos colectivos, así reciban allí otros nombres. En cierta forma, se pueden ver unas metodologías pedagógicas emergentes alejadas de los contenidos herméticos y más sintonizadas con el aprender creando, la ética DIY (en español, hágalo usted mismo), la creación con insumos propios y la remezcla con nuevos sentidos.

Segundo, se hace evidente su cercanía con las pedagogías críticas. Como se escribió líneas atrás, la emergencia de conceptos y prácticas asociadas a la educación expandida es de por sí una alteración al estado de cosas imperante y una crítica a la gramática de la modernidad. Además, con talleres formativos que buscan la elaboración de medios comunitarios artesanales (una radio, por ejemplo) o el conocimiento crítico de las caras menos amigables de internet (el internet profundo o la sensibilidad de los datos privados en línea) se busca un distanciamiento de lo meramente instrumental y estimular el pensamiento crítico con respecto al ecosistema digital, al tiempo que se aboga por prácticas educativas liberadoras de corte político en micro espacios ciudadanos tales como los barrios. 
Tercero, algunos ven la educación expandida como una pedagogía emergente. No es ese mi caso. Si se apela a la concepción normativa de la pedagogía, en el sentido de asumir el reto teórico y práctico de decir cómo debería ser la educación y, sobre todo, cómo hacer para que sea así, la educación expandida se queda corta a pesar de esbozar, sin orden sistemático, algunas ideas interesantes. Prefiero verla como un discurso crítico/práctico sobre educación, sin compromiso normativo ni prescriptivo, que busca provocar y estimular el debate al tiempo que pone en práctica una serie de presupuestos logísticos perfectamente susceptibles de cambio, mejora o abandono. Creo que visualizar/ explicitar unos lineamientos pedagógicos teóricos y metodológicos de diversa índole, tales como el aprendizaje entre pares o las prácticas formativas no vicarias, no implica abogar por una teoría pedagógica en concreto y menos sostener que son los pasos iniciales para la construcción de una Pedagogía, así con mayúscula. Se puede adelantar que en las prácticas de educación expandida hay elementos claramente contenidos en ideas pedagógicas de antaño y otros que inicialmente podrían catalogarse como emergentes, o sea, no beben de una sola fuente conceptual claramente delimitada, sino de múltiples grifos.

\section{Referencias}

Adell Segura, J., y Castañeda Quintero, L. (2012). Tecnologías emergentes, ¿pedagogías emergentes? En J. Hernández Ortega, M. Pennesi Fruscio, D. Sobrino López, y A. Vázquez Gutiérrez (Coords.), Tendencias emergentes en educación con TIC (pp. 13-32). Barcelona: Asociación Espiral, Educación y Tecnología.

Adell Segura, J., y Castañeda Quintero, L. J. (2015). Las pedagogías escolares emergentes. Cuadernos de Pedagogía, 462, 21-25.

Area Moreira, M. (2015). Reinventar la escuela en la sociedad digital. Del aprender repitiendo al aprender creando. En M. Poggi (Ed.), Mejorar los aprendizajes en la educación obligatoria. Políticas y actores (p. 167-194.). Buenos Aires: IIPE-Unesco.

Ayuste González, A., y Trilla Bernet, J. (2005). Pedagogías de la modernidad y discursos postmodernos sobre la educación. Revista de Educación, (336), 219-248.

Carbonell Sebarroja, J. (2015). Pedagogías del siglo XXI: alternativas para la innovación educativa. Barcelona: Octaedro.

Dewey, J. (2004). Democracia y educación: una introducción a la filosofía de la educación. Madrid: Morata.

Díaz, R., Gil, F. G., y Jiménez, P. (2010). Liberar el código fuente. Razón y Palabra. Cultura digital y vida cotidiana en Iberoamérica. Una revisión crítica más allá de la Comunicación, 15(73).

Díaz López, R. (2009). Educación expandida: la red como fuente de conocimientos. Periférica: Revista para el análisis de la cultura y el territorio, 10, 197-205.

Díaz López, R. (2012). Expanding education so that we can stop feeling that we need to be taught. En Expanded Education (english edition) (pp. 43-60). Sevilla: ZEMOS98. Recuperado a partir de http:// www.international.zemos98.org/IMG/pdf/expanded_education.pdf 
Encyclopaedia Britannica (2009). Alternative education. Recuperado de https://www.britannica.com/ topic/alternative-education

Fernández Enguita, M. (2013). El aprendizaje difuso y el declive de la institución escolar. RASE: Revista de la Asociación de Sociología de la Educación, 6(2), 150-167.

Fonseca Díaz, A. D. (2012). Metodologías abiertas, creación colectiva y transdisciplinariedad. Itinerario Educativo, 26(59), 115-141.

Fonseca Díaz, A. D., y Rueda Ortiz, R. (2012). Subjetividades, ciudadanías y tecnologías digitales. Diálogos de la Comunicación. Usos y prácticas de la comunicación, (84), 1-25.

Freinet, C. (1996). Técnicas Freinet de la escuela moderna. (J. Campos, Trad.). México: Siglo Veintiuno.

Freire, J. (2012). Educación expandida y nuevas instituciones: ¿es posible la transformación? En R. Díaz y J. Freire (Eds.), Educación Expandida (pp. 67-85). Sevilla: ZEMOS98.

Freire, P. (2005). Pedagogía del oprimido. (J. Mellado, Trad.). México, D.F.: Siglo XXI Editores.

Freire, P. (2011). La educación como práctica de la libertad. (L. Ronzoni, Trad.). México: Siglo XXI Editores. Godotti, M. (1998). Historia de las ideas pedagógicas. México: Siglo XXI.

González Monteagudo, J. (1988). Célestin Freinet, un precursor de la investigación en la escuela. Aspectos generales de su didáctica. Investigación en la Escuela, 6, 51-61.

ICEM (2000, junio). La charte de l'école moderne. Le Nouvel Educateur (120), 38-39. Recuperado de https://www.icem-freinet.fr/archives/ne/ne/120/charte120-pdf.pdf

Imbernón Muñoz, F. (2001). Célestin Freinet y la cooperación educativa. En J. Trilla Bernet (Ed.), El legado pedagógico del siglo XX para la escuela del siglo XXI (pp. 249-270). Barcelona: Graó.

Laudo Castillo, X. (2011). La hipótesis de la pedagogía postmoderna. Educación, verdad y relativismo. Teoría de la Educación. Revista Interuniversitaria, 2(23), 45-68.

Legrand, L. (1993). Célestin Freinet, un creador comprometido al servicio de la escuela popular (18961966). Perspectivas: revista trimestral de educación comparada, XXIII(1-2), 425-441.

Marín Ibañez, R. (1976). Los ideales de la Escuela Nueva. Revista de Educación, 242, 23-42.

Peña Sánchez, J., Noreña Noreña, N. M., Fonseca, A., del Pilar Navas, M., Fagua Fagua, A. P., y Fernández Jaimes, C. (2011). Una revisión de los procesos sociocognitivos y creativos en la educación mediada por los entornos digitales. Tesis psicológica: Revista de la Facultad de Psicología, 6, 14-35.

Prats, E. (2013). Jugando a ser dioses: puntos críticos de las tecnologías en educación. TESI, 14(3), 101-120.

Ricaurte, P. (2015, abril 24). Glosario de pedagogías emergentes [Entrada de blog]. Cultura digital. Recuperado de https://paolaricaurte.wordpress.com/2015/04/24/glosario-de-pedagogias-emergentes/

Rosan Boch (s.f.). [website]. Recuperado de http://www.rosanbosch.com/es

Sennett, R. (2009). El artesano. Barcelona: Anagrama.

Sharples, M., Adams, A., Alozie, N., Ferguson, R., FitzGerald, E., Gaved, M., ... Yarnall, L. (2015). Innovating Pedagogy 2015. Open University Innovation Report No. 4. Milton Keynes: The Open University. Recuperado a partir de http://oro.open.ac.uk/45319/1/InnovatingPedagogy_2015.pdf 
Trilla Bernet, J. (Ed.). (2001). El legado pedagógico del siglo XX para la escuela del siglo XXI. Barcelona: Graó.

Uribe Zapata, A. (2017a). Protoideas educativas de la educación expandida. Revista Virtual Universidad Católica del Norte, 51, 292-310. Recuperado a partir de http://revistavirtual.ucn.edu.co/index.php/ RevistaUCN/article/view/856/1374

Uribe Zapata, A. (2017b). Una revisión de prácticas educativas expandidas de la ciudad de Medellín. Rev. Guillermo de Ockham, 15(1), 139-148. http://dx.doi.org/10.21500/22563202.2757

Uribe Zapata, A. (2018). Concepto y prácticas de educación expandida: una revisión de la literatura académica. El Ágora USB, 18(1), 277-292. http://dx.doi.org/10.21500/16578031.3456

Veletsianos, G. (2010). A Definition of Emerging Technologies for Education. En G. Veletsianos (Ed.), Emerging technologies in distance education (pp. 3-22). Edmonton: AU Press. Recuperado a partir de http://www.aupress.ca/index.php/books/120177 